\title{
Н.С. Нечеухина
}

\section{РОЛЬ КОНТРОЛЛИНГА ПРИ РЕАЛИЗАЦИИ СТРАТЕГИИ РАЗВИТИЯ ПРЕДПРИЯТИЙ В УСЛОВИЯХ АВТОМАТИЗАЦИИ}

\author{
N.S. Necheukhina \\ THE ROLE OF CONTROLLING WHILE IMPLEMENTING \\ THE DEVELOPMENT STRATEGY OF THE ENTERPRISES \\ UNDER THE CONDITIONS OF AUTOMATION
}

\begin{abstract}
Рассматриваются основные вопросы развития контроллинга в современных условиях. Доказывается, что основу системы контроллинга составляет информация о деятельности предприятия и состоянии рыночной среды его функционирования, регулярный и своевременный сбор которой, ее анализ и планирование приобретают первостепенное значение. Особая роль в статье отводится стратегическому контроллингу, рассматриваются различные точки зрения на данную проблему. Существенное внимание уделяется подготовке информации руководителю, значимых показателей финансового плана (бюджета) и разработке мероприятий для реализации заданных целей. Применение организационной и компьютерной техники в учетном процессе контроллинга превращает ее в непосредственный организационноэкономический инструмент учета. Многообразие различных подходов и трактовок контроллинга показывает на сложность выработки единого методологического подхода к исследованию контроллинга. С учетом многогранности контроллинга и его эффективного влияния на управление предприятием дается авторская трактовка контроллинга. Проведенное исследование понятия «контроллинг», широко используемое в зарубежной и отечественной экономической литературе, применительно к промышленным предприятиям выявило, что современный контроллинг - необходимый элемент менеджмента. Для эффективности принимаемых управленческих решений необходимо не только обеспечить поступление информации, отражающей реальное положение дел, организовать унифицированную, сжатую, четкую и простую форму ее подачи, но и соблюсти своевременность ее поступления. Только оперативность сбора и обработки данных, их значимость и актуальность позволяют принимать эффективные бизнесрешения. И здесь центральное место занимает система учета промышленного предприятия, в частности уровень ее развития. С увеличением объемов информации, необходимой для принятия управленческих решений, и сокращением сроков на ее предоставление соответственно возрастает число событий хозяйственной деятельности предприятия, требующих учета. В связи с этим разработка и реализация теоретических положений и практических рекомендаций по моделированию учетных событий в системе контроллинга промышленного предприятия, обеспечивающих успешное функционирование предприятия в долгосрочном периоде, является важнейшей задачей современного менеджмента.

СТРАТЕГИЧЕСКИЕ РЕШЕНИЯ; ЭФФЕКТИВНОЕ УПРАВЛЕНИЕ; МЕНЕДЖМЕНТ; РАЗВИТИЕ КОНТРОЛЛИНГА; РЫНОЧНАЯ СРЕДА; УПРАВЛЕНЧЕСКИЕ РЕШЕНИЯ; УПРАВЛЕНИЕ ПРЕДПРИЯТИЕМ; АВТОМАТИЗАЦИЯ УЧЕТНЫХ СОБЫТИЙ; ПРОГРАММНОЕ ОБЕСПЕЧЕНИЕ КОНТРОЛЛИНГА.
\end{abstract}

The article considers the main issues of the development of controlling in the current context. It is proved that the principal of the controlling system based on the information about company's activities and the status of the market environment. A regular and timely collection of this information, its analysis and planning is of primary concern. A special attention is paid to the strategic controlling and various points of view on this issue. The article focuses considerable attention on the information prepared for the management, significant indicators of the financial plan (budget) and the development of measures to implement goals. Implementing organizational and computer technology in the accounting process of controlling turns it into a close organizational and economic accounting tool. The variety of different approaches and interpretations of controlling shows the complexity of developing a unified methodological approach to the study of controlling. Taking into account the complexity of controlling and its effective influence on the management of the enterprise, the article provides the author's interpretation of controlling. A conducted study of controlling, widely used in foreign and domestic economic literature, with regard to industrial enterprises has revealed that modern controlling is an essential element of modern management. The article discusses various approaches to 
implement the software controlling the complex system and application software, ensuring the implementation of the broad range of work on the automation of accounting events. An integrated operation of all computer devices and their interaction with the controller provide a software workstation controller, which is divided into general and functional. The article underlines their similarities and differences. Thus, the development and implementation of theoretical principals and practical guidance for modelling accounting events in the controlling system of the enterprise that provide successful functioning in the long term is the most important task in modern management.

STRATEGIC DECISIONS; EFFECTIVE MANAGEMENT; MANAGEMENT; DEVELOPMENT OF CONTROLLING; MARKET ENVIRONMENT; MANAGEMENT SOLUTIONS; ENTERPRISE MANAGEMENT; AUTOMATION OF ACCOUNTING OF EVENTS; THE SOFTWARE CONTROLLING.

Введение. Для эффективности принимаемых управленческих решений необходимо не только обеспечить поступление информации, отражающей реальное положение дел, организовать унифицированную, сжатую, четкую и простую форму ее подачи, но и соблюсти своевременность ее поступления. Только оперативность сбора и обработки данных, их значимость и актуальность позволяют принимать эффективные бизнес-решения. И здесь центральное место занимает система учета промышленного предприятия, в частности уровень ее развития. С увеличением объемов информации, необходимой для принятия управленческих решений и сокращением сроков на ее предоставление, соответственно возрастает число событий хозяйственной деятельности предприятия, требующих учета.

В связи с этим, разработка и реализация теоретических положений и практических рекомендаций по моделированию учетных событий в системе контроллинга промышленного предприятия, обеспечивающих успешное функционирование предприятия в долгосрочном периоде, является важнейшей задачей современного менеджмента.

Методика исследования. Для эффективного менеджмента промышленным предприятием, его устойчивым развитием в условиях рисков и неопределенности конкурентной среды должна быть разработана система контроллинга. Чтобы поставить контроллинг на службу поступательного развития экономики предприятия, необходимо развивать его теорию и методологию, повышать уровень системной организации и координации всего процесса менеджмента.

Существует множество трактовок контроллинга, которые даются ведущими учеными и экономистами в научно-популярных изданиях, монографиях, пособиях.
Следует отметить Т. Райхмана [15], который контроллингом называет систему, ориентированную на результат с учетом обеспечения ликвидности, в сферу задач которой входит сбор и обработка информации в процессе разработки, координации и контроля за выполнением планов на предприятии.

В.А. Анташов и Г.В. Уварова рассматривают контроллинг как определенную концепцию руководства предприятием, ориентированную на его долговременное и эффективное функционирование в постоянно меняющихся хозяйственных условиях [2].

А.П. Градов под контроллингом понимает новейшую концепцию эффективного управления фирмой для обеспечения ее долгосрочного сушествования на рынке. Это система обеспечения выживаемости предприятия на этапах стратегического и тактического управления [3].

В.Б. Ивашкевич [4] трактует контроллинг как систему управления прибылью предприятия для достижения ее максимизации.

Определения контроллинга, предлагаемые разными учеными, кажутся с первого раза однообразными и повторяющимися, но отличаются по их контексту и той концепции, которой они придерживаются и вследствие которой значительно отличаются цели и задачи контроллера при различном толковании данного понятия.

Основу системы контроллинга составляет информация о деятельности предприятия и состоянии рыночной среды его функционирования, регулярный и своевременный сбор которой, ее анализ и планирование приобретают первостепенное значение в современных российских реалиях. В связи с этим возрастает интенсивность и насыщенность информационных потоков, требующих систематизации, унификации для обеспечения эффективности процессов управления бизнесом. 
На наиболее существенные задачи стратегического контроллинга указывают Ю.П. Анискин, А.М. Павлова [1]. Контроллинг на сегодня представляет собой один из лучших систематизаторов масштабных потоков информации о движении экономической информации в рамках промышленных предприятий с целью координации принимаемых управленческих решений.

Контроллинг нельзя отождествлять с контролем и ревизией. В системе контролинга выделяется оперативный контроль, который занимается вопросами учета и анализа затрат, результатов хозяйственной и финансовой деятельности. Анализ базируется на данных бухгалтерской отчетности. Плановая служба представляет статистические выкладки, плановые данные и результаты сравнения фактических значений показателей. С.Г. Фалько [16] к основным задачам стратегического контроллинга относит консультирование менеджеров и собственников предприятия при выработке генеральной стратегии, стратегических целей и мероприятий по направлениям деятельности, а также предоставление необходимой информации для принятия стратегических решений.

Задача контролера на базе анализа дать предложение (рекомендации) руководителю по сокращению затрат и увеличению прибыльности (рентабельности) производства и сбыта.

При сложном производстве контроллинг разбивается по технологической цепочке. Так, при наличии дочерних предприятий ответственность за конечные финансовые результаты и функции оперативного управления делегированы дочерним предприятиям, а прибыль внутри разбивается по подразделениям (внутренний хозрасчет). В ведении центров ответственности затрат находятся денежные потоки, инвестиции, контроль за стратегическими целями предприятия, так как отдельные подразделения могут в своей деятельности расходиться со стратегическими задачами головного предприятия. М.Н. Павленков [10] считает, что контроллинг - это межфункциональная система (подсистема) предприятия, являющаяся составной частью общей системы управления, основополагающими понятиями которой являются закономерности, принципы, функции, цели, задачи, методы.
Контроллинг начинается с анализа производственных и сбытовых затрат подразделениями - центрами ответственности. Бухгалтер-контролер может обладать правом «вето» при принятии многих управленческих решений, таких как: обоснование цены; новые инвестиции; покупка и производство новых товаров и изделий; формирование производственной ассортиментной структуры. Существенное внимание уделяется подготовке информации руководителю, значимых показателей финансового плана (бюджета) и разработке мероприятий для реализации заданных целей [12]. Особое место отводится конъюнктурным решениям вопроса, сведениям о конкурентах и т. Д. С этой целью предоставляется прогнозная финансовая оценка мероприятий. Оперативно анализируются:

- ликвидность предприятия;

- отклонение плановых величин от факта, причины;

- прогноз развития событий;

- мероприятия по снижению затрат.

Для решения данных и других задач рекомендуется: разработать систему внутренней отчетности и специальный программный продукт; рассчитать точку безубыточности; произвести анализ зависимостей между объемом производства, себестоимостью, прибылью, трудовыми затратами и т. д. С этой целью ежемесячно составляется и представляется менеджеру отчетность, а именно: статистика объемов выпуска; расчет издержек производства и обращения; исчисление финансовых результатов по различным схемам учета затрат; расчет суммы покрытий (маржинального дохода); расчет показателей рентабельности; расчет ликвидности; статистика движения и использования рабочей силы; статистика движения запасов; расчет денежных потоков; инвестиционные расчеты; и т. д.

Интерес к комплексному определению контроллинга в России наметился в последние 5-7 лет в трудах таких известных российских специалистов, как А.М. Карминский, С.Г. Фалько [16]. Согласно их мнению, контроллинг должен иметь четыре составляющие - философскую, функциональную, организационную и научную.

Контроллинг при реализации стратегии развития предприятия рассматривается многими авторами в нескольких направлениях. 
Первая группа концепций - концепции с ориентацией на учет, которые

применяются в тех случаях, когда преследуются информационные цели, которые могут быть реализованы с помощью данных бухгалтерского учета. Внимание концентрируется на обеспечении прибыли всей организации, несмотря на различия в целях хозяйственных областей и отдельных сотрудников. Представителем данной концепции является Д. Шнайдер [18].

Концепция, ориентированная на систему учета, в качестве основной и единственной сферы деятельности контроллинга рассматривает систему учета. При этом под системой учета понимают специфическую информационную систему, которая позволяет руководству в любой момент времени получать необходимые для планирования и контроля количественные данные о деятельности предприятия. Основными задачами контроллинга являются централизация и переориентация в будущее всей системы учета, сконцентрированной на регистрации фактической информации прошлых периодов. В этой концепции реализуется лишь один элемент контроллинга, что не позволяет полностью использовать его потенциал. Такой подход актуален на начальной стадии внедрения контроллинга [8].

Одной из концепций, получивших наибольшее распространение, является концепция, ориентированная на управленческий учет. Данная концепция рассматривает контроллинг как совокупность организационных мероприятий по совершенствованию документооборота в рамках управленческого учета. Здесь явно прослеживается ориентация на управление затратами с использованием информационных технологий.

Этот подход может быть охарактеризован как ориентированный на прибыль или ограниченно ориентированный на информацию, поскольку основывается только на данных бухгалтерского учета. Базирующийся на учете контроллинг охватывает релевантные состоянию дел данные в денежном выражении. Эта количественность задается преимушественно в оперативных связях. Поэтому контроллинг с данной точки зрения относится, в первую очередь, к оперативному уровню и лишь отчасти к стратегическому, когда речь заходит о потенциале успеха. Д. Шнейдер вообще предлагает ограничить контроллинг вспомогательными контрольными и координационными задачами на базе данных бухучета. Однако ограничение его чисто денежными показателями представляется слишком узким подходом.

O концепциях с ориентацией на учет можно говорить в тех случаях, когда преследуются информационные цели, которые могут быть реализованы, в первую очередь, с помощью данных бухгалтерского учета. Внимание при этом концентрируется на показателях успеха в денежном выражении. Речь идет, прежде всего, об обеспечении прибыли всего предприятия, несмотря на различия в целях хозяйственных областей и отдельных сотрудников.

Приверженцы этой концепции являются последователями немецкой школы, основные положения которой приведены в известных переводных работах Р. Манна и Э. Майера [6, 7], Х. Фольмута [17].

Благодаря тому что концепция с ориентацией на управленческий учет предлагалась одной из первых и развивалась в течение двух десятилетий, она на сегодняшний день, пожалуй, наиболее популярна. Востребованность знаний в области контроллинга и управленческого учета подтверждается появлением многочисленных программ на рынке бизнес-образования.

В литературных источниках, ориентированных на данную концепцию контроллинга, по сути, описывается управленческий учет и приводятся методы и модели управления затратами и бюджетирования [13].

Концепции с ориентацией на информацию открывают более широкую перспективу. Они выходят за рамки подхода, ориентированного на бухгалтерский учет, и охватывают всю целевую систему предприятия, включая не только денежные величины. В соответствии с данными концепциями наряду с бухгалтерской используется количественная и качественная информация, причем ее источником являются непосредственно хозяйственные области организации. Представителем данной концепции является Т. Райхманн [15], который считает основной задачей контроллинга обеспечение руководства организации информацией для принятия решений [11].

Главная задача контроллинга здесь видится в координации получения и подготов- 
ки информации с потребностями в ней. В соответствии с этой концепцией контроллинг - это выходящий за рамки одной функции управленческий инструмент, который поддерживает внутрифирменный процесс управления и принятия решений с помощью целенаправленного подбора и обработки информации.

Однако рациональное включение столь обширных информационных задач в хозяйственную структуру предприятия представляет трудную проблему. Эти задачи не самоцель, они являются производными вышестоящих целей, которые связаны со специфическими информационными потребностями. Если вся область информационного хозяйства становится функцией контроллинга, то ее включение в общефирменное поле действия приобретает неопределенный, размытый характер, поскольку информационные потребности вышестоящих инстанций слишком многоаспектны и слабо специфицированы. В этом изъян данной группы концепций.

Различие между концепциями первой и второй групп кроется в уровне принятия решений. В первом случае приоритет отдается оперативным задачам, при этом информационной основой служат количественные данные бухгалтерского и управленческого учета, во втором случае информационная база принятия решений расширяется за счет данных функциональных областей, а уровень принятия решений приближается к стратегическому.

Рационализация принятия решений и использование инструментов, характерные для количественного подхода к управлению, повысили роль управленческих информационных систем. Это стимулировало появление и развитие данной концепции контроллинга, акцентирующей внимание на информационном обеспечении.

На эффективность учета наибольшее влияние оказывают информационные технологии, которые на базе вычислительной и организационной техники, создания автоматизированного рабочего места контроллера (АРМ контроллера) позволяют повысить уровень автоматизации функций контроллера и органически обеспечить вход в единую интегрированную информационную систему управления предприятием.
Применение организационной и компьютерной техники в учетном процессе контроллинга превращает ее в непосредственный организационно-экономический инструмент учета. Основу технического комплекса учетных технологий составляют средства компьютерной техники: персональные компьютеры, серверы определенного типа, средства коммуникационной техники, организационная техника, а также программное обеспечение в составе комплекса системных и прикладных программ, обеспечивающих реализацию всего комплекса работ по автоматизации учета.

Для успешного функционирования развития автоматизации учета в системе контроллинга необходимо выделить несколько этапов.

Первый этап - в группе учета данных службы главного контроллера создание автоматизированных рабочих мест контроллеров. На организационно-функциональной стадии АРМ контроллера функционирует в автономном режиме с использованием локальной учетной базы данных [9].

Согласованную работу всех устройств ПЭВМ и их взаимодействие с контроллером обеспечивает программное обеспечение АРМ контроллера, которое подразделяется на общее и функциональное. При создании АРМ контроллера в качестве базовых используются программные средства:

- для подготовки текстов - текстовые редакторы или текстовые процессоры;

- для подготовки табличных документов табличные процессоры или электронные ведомости;

- для автоматизации работ по созданию и ведению учетных баз данных - системы управления базами данных.

Большое распространение получили интегрированные пакеты функционального программного обеспечения, включающие текстовый процессор, табличный процессор, системы управления базами данных, а также конкретный командный файл настройки программного обеспечения на конкретный вид и режим обработки информации. Это позволяет организовать работу контроллера на АРМ в режиме «non-stop» с максимальным учетом его профессиональных требований. 
АРМ контроллера на базе ПЭВМ является технико-технологическим средством освоения учетных информационных ресурсов предприятия, обусловливающим способность его успешного развития. В рамках АРМ контроллера весь учетный информационный фонд предприятия функционирует в форме:

- локальной учетной базы данных - фактографические данные о финансово-хозяйственной деятельности предприятия;

- базы знаний - подходы, принципы, методы и методика учета;

- программных средств - инструмент автоматизированного исполнения учетных задач для информационного обслуживания разработки и принятия управленческих решений менеджерами предприятия.

Второй этап развития автоматизации учета в системе контроллинга - интеграционный. Данный этап обеспечивает интеграцию локальных АРМ контроллера в единую интегрированную информационную систему промышленного предприятия. Вопросы интеграции АРМ контроллера в единую информационную систему выявили следующие основные проблемы, связанные с неэффективностью функционирования информационных систем промышленных предприятий:

- информационные системы промышленных предприятий не имеют научнометодического обеспечения, вследствие чего отсутствует концепция построения и развития, приводящая к отсутствию целостности. Информационные системы представляют собой набор малосвязанных между собой информационных ресурсов и процедур управления ими, что приводит к снижению эффективности управления предприятием;

- использование многообразных информационных технологий обработки и передачи информации, отсутствие единых стандартов хранения данных, отличие их от общемировых приводит к сдерживанию процессов интеграции информационных потоков между различными структурными подразделениями и уровнями управления предприятием;

- невысокий уровень автоматизации информационного процесса в структурных подразделениях предприятия приводит к несбалансированным управленческим решениям.
В ходе интеграции АРМ контроллера в информационную систему предприятия отлаживается механизм функционирования информационных технологий, обеспечивающий корректность, актуальность, сохранность и санкционированный доступ к учетной, плановой, статистической и другой информации о финансово-хозяйственной деятельности предприятия.

Третий этап - автоматизация учетных событий в системе контроллинга является перспективным, способным обеспечить правильное решение управленческой проблемы за счет прогнозирования и предвидения результата прогнозируемых управленческих событий в деятельности предприятия.

Автоматизация учетных событий в системе контроллинга с использованием интегрированных в единую информационную систему предприятия автоматизированных рабочих мест контроллеров обладает рядом существенных особенностей, связанных как с объектом моделирования, так и с применяемым аппаратом и средствами моделирования, а также с необходимостью определять количественные значения особо важных учетных событий в деятельности предприятия в режиме реального времени и по запросу.

Предложенный данный механизм функционирования задач учетного комплекса системы контроллинга основного производства консолидирует учетную информацию по изготовлению и выпуску продукции бизнеспроцессами и ее предоставление менеджерам для принятия своевременных управленческих решений по регулированию хода производства и обеспечению выпуска продукции в необходимых объемах и в установленные сроки.

Многообразие различных подходов и трактовок контроллинга показывает на сложность выработки единого методологического подхода к исследованию контроллинга.

Существующий практический опыт организации и функционирования контроллинга на промышленных предприятиях недостаточно обобщен и требует дальнейших исследований и рекомендаций по его совершенствованию и повышению эффективности.

Учитывая многогранность контроллинга и его эффективное влияние на управление 
предприятием, далее будем понимать контроллинг как управленческую систему, интегрирующую и координирующую все функциональные сферы деятельности для достижения оперативных и стратегических целей в рамках менеджмента предприятия [12].

Это определение контроллинга отражает:

1) контроллинг - управленческая система в рамках функционирующей на промышленном предприятии системы менеджмента;

2) система контроллинга состоит их двух функциональных подсистем: стратегический контроллинг и оперативный контроллинг, решающие свои цели и задачи;

3) контроллинг как эффективный механизм интеграции и координации всех функциональных сфер деятельности для достижения поставленных стратегических, тактических и оперативных целей, программ планов, задач развития предприятия и его структурных подразделений.

По своему функциональному содержанию и роли система контроллинга должна занимать центральное место в системе менеджмента промышленным предприятием (см. схему).
Научная новизна полученных результатов исследования заключается в разработке и обосновании теоретических положений формирования инструментария моделирования учетных событий как основы повышения эффективности контроллинга на предприятиях промышленности в условиях факторов неопределенности и рисков, обусловленных воздействием рыночной среды функционирования.

Разработанная система концептуальных моделей повышает эффективность системы контроллинга промышленного предприятия, его учетного комплекса и оценки их влияния на эффективность управления производственным процессом при своевременном обеспечении менеджеров полноценной учетной информацией.

Результаты исследования. В процессе исследования получены как теоретические, так и практические результаты:

- предложена авторская трактовка контроллинга как управленческой системы, интегрирующей и координирующей все функциональные сферы деятельности для достижения оперативных и стратегических целей в рамках менеджмента предприятия;

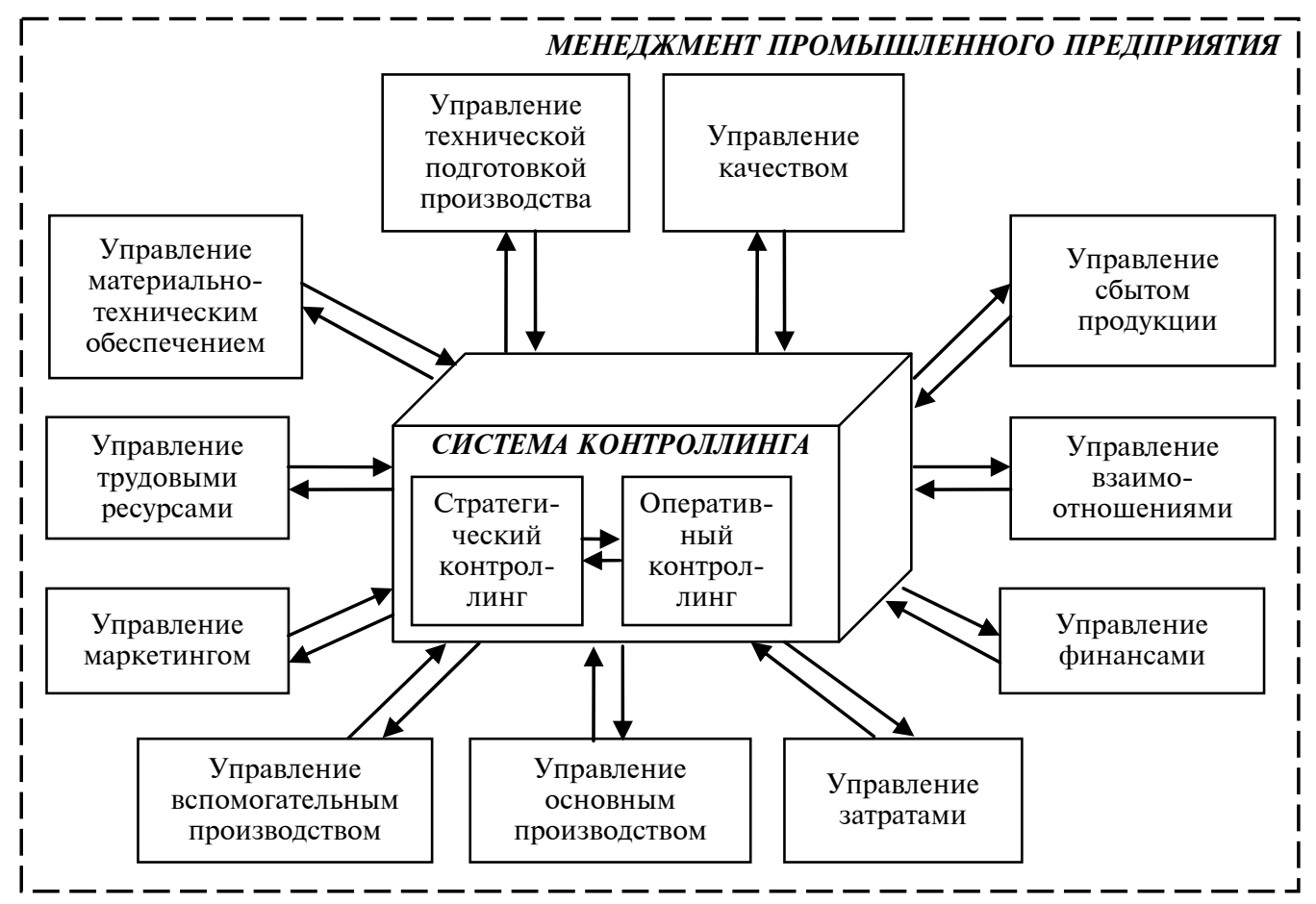

Место системы контроллинга в менеджменте промышленного предприятия 
- уточнен организационно-экономический механизм системы контроллинга, который существенно влияет на достижение поставленных оперативных и стратегических целей предприятия;

- обоснована концептуальная модель повышения эффективности системы контроллинга промышленного предприятия в условиях автоматизации;

- разработана модель системы контроллинга основного производства, состоящая из задач, в процессе функционирования которых обеспечивается информационная поддержка принятия своевременных и обоснованных управленческих решений;

- представлена концепция определения оценки влияния задач системы контроллинга на эффективность управления производственным процессом по ведущим бизнеспроцессам производства.

Bыводы. Практическая значимость проведенного исследования состоит в разработ- ке авторского понятия «Контроллинг» применительно к промышленным предприятиям, что позволяет утверждать: современный контроллинг - необходимый элемент менеджмента.

Эффективность финансово-хозяйственной деятельности предприятия, достижение оперативных, тактических и стратегических целей развития предприятия и его структурных подразделений в значительной степени зависят от качества и действенности контроллинга.

Направления дальнейших исследований заключаются в разработке методики внедрения системы контроллинга на предприятиях промышленности как передовой управленческой инновации, которая может служить фундаментальной составляющей организационных преобразований всей системы управления промышленного предприятия, направленных на повышение эффективности его функционирования в современных условиях.

\section{СПИСОК ЛИТЕРАТУРЫ}

1. Анискин Ю.П., Павлова А.М. Планирование и контроллинг. М.: Омега-Л, 2007. 280 с.

2. Анташов В.А., Уварова Г.В. Экономический советник менеджера. М.: АКДИ «Экономика и жизнь», 2009. 384 с.

3. Стратегия и тактика антикризисного управления фирмой / под общ. ред. А.П. Градова, Б.И. Кузина. СПб.: Спец. литература, 1996.

4. Ивашкевич В.Б. Контроллинг: сущность и назначение // Бухгалтерский учет и аудит. 1991 № 7. C. $8-12$.

5. Карминский А.М., Фалько С.Г., Жевага А.А., Иванова Н.Ю. Контроллинг: учебник / под ред. А.М. Карминского, С.Г. Фалько. М.: Финансы и статистика, 2006. 336 с.

6. Майер Э. Контроллинг как система мышления и управления: пер. с нем. / под ред. С.А. Николаевой. М.: Финансы и статистика, 1993. 96 с.

7. Манн Р., Майер Э. Контроллинг для начинающих. М.: Финансы и статистика, 1995. 304 с.

8. Набок Р., Набок А. Американская и немецкая модели контроллинга // Финансовый директор. 2007. № 12. URL: http://www.econfin.ru/ rus/ library/corpsystems/accmanage/index.php?6570

9. Нечеухина Н.С. Финансовый инструментарий формирования управленческого учета, контроллинга и анализа в структуре стратегического управления предприятием // Известия Уральского государственного экономического университета. 2008. № 3(22). С. 68-74

10. Павленков М.Н. Контроллинг промышленного предприятия: методология, теория, прак- тика: [моногр.]. Н. Новгород: Изд-во Волговятской академии гос. службы, 2007. 363 с.

11. Попченко Е.Л., Ермасова Н.Б. Бизнес-контроллинг. М.: Финансы и статистика, 2006. 288 с.

12. Пыткин А.Н. Перспективы развития промышленной политики // Совершенствование стратегического управления корпорациями и региональная инновационная политика : матер. рос науч.-практ. конф. с междунар. участием. Пермь, 2012. C. $142-146$.

13. Пыткин А.Н. Теория и методология взаимосвязи стратегического и оперативного контроллинга. Екатеринбург: Изд-во Ин-та экономики УрО РАН, 2009. 162 с.

14. Пыткин А.Н., Блаженкова Н.М., Власова И.Е Управленческий учет: теория и практика организации на промышленном предприятии: [моногр.]. Пермь: Институт экономики УрО РАН, 2005. 220 с

15. Райхман T. Менеджмент и контроллинг. Одни цели - разные пути и инструменты // Международный бухгалтерский учет. 1999. № 5. С. 26-36.

16. Фалько С.Г. Контроллинг для руководителей и специалистов. М.: Финансы и статистика, 2008. 272 c

17. Фольмут Х.Й. Инструменты контроллинга от А до Я: пер. с нем. / под ред. и с предисл. М.Л. Лукашевича и Е.Н. Тихоненковой. М.: Финансы и статистика, 2003. 288 с.

18. Шнайдер Д. Контроллинг как инструмент управления предприятием / Е.А. Ананькина, С.В. Данилочкин, Н.Г. Данилочкина [и др.]; под ред. Н.Г. Данилочкиной. М.: Аудит, Юнити, 2003. 279 с. 


\section{REFERENCES}

1. Aniskin IuP, Pavlova AM Planning and controlling. Moscow, Omega-L, 2007. 280 p. (rus)

2. Antashov V.A., Uvarov G.V. Economic Advisor to the manager. Moscow, ACDI «Economics and Life», 2009. 384 p. (rus)

3. Strategy and tactics of anti-crisis management firm. Ed. by A.P. Gradova, B.I. Cousin. St. Petersburg, Special literature, 1996. (rus)

4. Ivashkevich V.B. Controlling: the nature and purpose. Accounting and auditing, 1991, no. 7, pp. 812. (rus)

5. Karminskii A.M., Fal'ko S.G., Zhevaga A.A., Ivanova N.Iu. Controlling: the textbook. Ed. A.M. Karminskogo, S.G. Falko. Moscow, Finance and Statistics, 2006. 336 p. (rus)

6. Mayer E. Controlling the system thinking and management: Per. with it. Ed. S.A. Nikolaeva. Moscow, Finance and Statistics, 1993. 96 p. (rus)

7. Mann R., Meyer E. Controlling for beginners. Moscow, Finance and Statistics, 1995. 304 p. (rus)

8. Nabok R., Nabok A. American and German models of controlling. CFO, 2007, no. 12. URL: http://www.econfin.ru/rus/library/corpsystems/accma nage/index.php?6570 (rus)

9. Necheuhina N.S. Financial instruments form of management accounting, controlling and analyzing the structure of strategic management. Proceedings of the Ural State Economic University, 2008, no. 3(22), pp. 68-74. (rus)

10. Pavlenkov M.N. Controlling industrial enterprises: methodology, theory, practice: Monograph. Nizhny Novgorod, Publishing House of the Volga-Vyatka
Academy of State Service, 2007. 363 p. (rus)

11. Popchenko E.L, Ermasova N.B. Business Controlling. Moscow, Finance and Statistics, 2006. 288 p. (rus)

12. Pytkin A.N. Prospects for the development of industrial policy. Improving the strategic management corporations and regional innovation policy: the materials of the Russian scientific-practical conference with international participation. Perm, 2012, pp. 142-146. (rus)

13. Pytkin A.N. Theory and Methodology of the relationship of strategic and operational controlling. Ekaterinburg: Publishing House of the Institute of Economics of UB RAS, 2009. 162 p. (rus)

14. Pytkin A.N., Blazhenkova N.M., Vlasov I.E. Management accounting: theory and practice of the organization in an industrial plant: monograph. Perm, Institute of Economics, Ural Branch of Russian Academy of Sciences, 2005. 220 p. (rus)

15. Reichman T. Management and Controlling. One goal - different ways and tools. International accounting, 1999 , no. 5, pp. 26-36. (rus)

16. Falco S.G. Controlling for managers and professionals. Moscow, Finance and Statistics, 2008. 272 p. (rus)

17. Folmut H.J. Controlling Instruments from A to Z: lane. with it. Ed. and foreword. M.L. Lukashevicha, E.N. Tihonenkovoy. Moscow, The Finance and statistics, 2003. 288 p.

18. Schneider D. Controlling as a management tool now. E.A. Anankina, S.V. Danilochkina, N.G. Danilochkina [et al.]. Ed. NG Danilochkina. Moscow, Audit, Unity, 2003. 279 p. (rus)

НЕЧЕУХИНА Надежда Семеновна - заведующий кафедрой «Бухгалтерский учет и аудит» Уральского государственного экономического университета, доктор экономических наук.

620144, ул. 8 Марта/Народной воли, д. 62/45, г. Екатеринбург, Россия. E-mail: nnecheuhina@yandex.ru

NECHEUKHINA Nadezhda S. - Ural State Economic University.

620144. 8 March str. 62/45. Ekaterinburg. Russia. E-mail: nnecheuhina@yandex.ru 